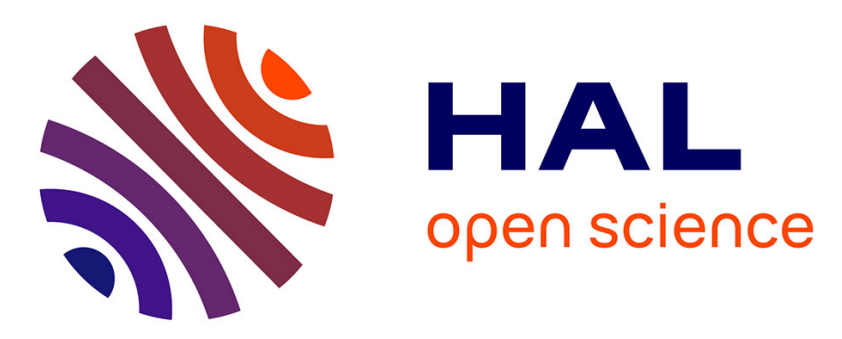

\title{
Olfactory anhedonia and negative olfactory alliesthesia in depressed patients
}

\author{
Boriana Atanasova, Wissam El-Hage, Claire Chabanet, Philippe Gaillard, \\ Catherine Belzung, Vincent Camus
}

\section{- To cite this version:}

Boriana Atanasova, Wissam El-Hage, Claire Chabanet, Philippe Gaillard, Catherine Belzung, et al.. Olfactory anhedonia and negative olfactory alliesthesia in depressed patients. Psychiatry Research, 2010, 176 (2-3), pp.190-196. 10.1016/j.psychres.2008.11.016 . hal-02526735

\section{HAL Id: hal-02526735}

\section{https://hal-univ-tours.archives-ouvertes.fr/hal-02526735}

Submitted on 31 Mar 2020

HAL is a multi-disciplinary open access archive for the deposit and dissemination of scientific research documents, whether they are published or not. The documents may come from teaching and research institutions in France or abroad, or from public or private research centers.
L'archive ouverte pluridisciplinaire HAL, est destinée au dépôt et à la diffusion de documents scientifiques de niveau recherche, publiés ou non, émanant des établissements d'enseignement et de recherche français ou étrangers, des laboratoires publics ou privés. 
Atanasova B, El-Hage W, Chabanet C, Gaillard P, Belzung C, Camus V. Olfactory anhedonia and negative olfactory alliesthesia in depressed patients. Psychiatry Research 2010;176(2-3):190-196. doi:10.1016/j.psychres.2008.11.016

\title{
Olfactory anhedonia and negative olfactory alliesthesia in depressed patients
}

\author{
Boriana Atanasova a,b,* Wissam El-Hage ${ }^{\text {a,c }}$, Claire Chabanet ${ }^{\mathrm{d}}$, Philippe Gaillard ${ }^{\text {a,c }}$, \\ Catherine Belzung ${ }^{\text {a }}$, Vincent Camus ${ }^{\mathrm{a}, \mathrm{c}}$ \\ ${ }^{a}$ INSERM U930 \& IFR 135, Université François Rabelais, Parc Grandmont, 37200 Tours, France, Tel: +33 (0)2 \\ 473674 38, E-mail: atanasova@univ-tours.fr \\ ${ }^{\mathrm{b}}$ Département Sensoriel et Innovation, Université François Rabelais, 37200 Tours, France \\ ${ }^{c}$ Clinique Psychiatrique Universitaire, CHRU de Tours, 37044 Tours Cedex 01, France \\ ${ }^{\mathrm{d}}$ Unité Mixte de Recherches FLAVIC, INRA/ENESAD, 21065 Dijon Cedex, France
}

\begin{abstract}
Anhedonia is a core symptom of depression. The present study aimed to investigate an "olfactory anhedonia" in depressed patients. Two odorants, one with pleasant (vanillin), and one with unpleasant (butyric acid) hedonic valence were evaluated by 30 depressed inpatients and 30 controls (healthy subjects, matched by age and gender). Participants explored the hedonic valence, intensity (discrimination) and perceived quality (identification) of 16 different stimuli (3 concentrations of odorants, their 9 combinations and one control containing distilled water). The hedonic perception showed that patients perceived unpleasant odorant significantly more unpleasant than controls. Concerning the intensity ratings, controls succeeded to discriminate between all concentrations of odorants, while patients discriminated between the different concentrations only for the unpleasant component. Regarding the identification task, patients perceived significantly more the unpleasant odorant than the pleasant one in an iso-intense unmixed odorants mixture, whereas controls perceived equally both odorants. These results support the notion of an olfactory perception impairment in depression. Further studies are needed to replicate these findings and to confirm the olfactory anhedonia or the olfactory negative alliesthesia in depressed patients.

Keywords. Depression; Olfactory perception; Binary mixtures; Hedonic valence
\end{abstract}

\section{Introduction}

Anhedonia, or failure to gain pleasure from normally pleasant experiences, is a marker for depressive state (Klein, 1974). Cognitive theories of depression claim that major depressive disorder (MDD) is associated with a systematic negative bias in thinking, negative view of self, of experiences, and of the future (Wright and Beck, 1983). This negatively biased associative processing impact a wide range of mental processes like memory, attention, thought process, decision making, language, executive and motor functions. Representations that rule actions, goal directed behaviours and adaptation to the environment emerge from cognitive activity that roots into sensorial (Mesulan, 1998) and emotional perceptions (Phillips et al., 2003). Indeed, MDD patients show an increased sensitivity to recognition and recall of negative stimuli (Mathews and MacLeod, 1994) and a reduced anticipation for positive experiences (MacLeod and Byrne, 1996). However, researchers on cognitive dysfunction in depression have mainly focused on processes supported by visual or verbal inputs. Less has been done on cognitive functions that are underlined by olfactory stimuli. Even so, olfactory processing has been shown to have close links with emotional processing (Ehrlichman and Bastone, 1992; Zatorre et al., 2000).

The application of modern brain imaging techniques confirmed that odours and emotions are processed in similar brain structures: the orbitofrontal and insular cortex, the hypothalamus, and the amygdala (Zald and Pardo, 2000; Lane et al., 1997). Moreover, a number of studies 
have indicated that odours can induce positive and negative affects which can modulate mood (Schiffman et al., 1995a; Schiffman et al., 1995b), behaviour (Millot and Brand, 2001), cognition (Gilbert et al., 1997; Broughan, 2002), autonomic parameters (Nagai et al., 2000) and cerebral activity measured by electrophysiological recording (Brand, 1999) and neuroimaging (Brand, 1999; Royet et al., 2003).

It has been shown that odour perception in depression is altered at a peripheral level (odour detection threshold) and possibly at a central level (identification, discrimination, memory or the ability to label an odour) (Serby et al., 1990; Rupp et al., 1997; Pause et al., 2001; Lombion-Pouthier et al., 2006). Studies exploring this olfactory deficit have presumed that such alterations may be related to an overlap in brain structures involved in the processing of olfactory or emotional cues and structures involved in depression. For instance, previous studies have found that depressive patients differed from controls in the emotional evaluation of odours (Steiner et al., 1993; Pause et al., 2000). Thus, Steiner et al. (1993) found that the facial reactions of depressive patients to pleasant and unpleasant odours were significantly shorter than those of controls. Pause et al. (2000) showed that MDD patients responded with higher arousal to negative stimuli (odours and emotional pictures) than healthy controls. Thus, one could suppose that MDD patients would display an impaired olfactory perception of pleasant odorants compared to unpleasant odorants. Relationship between clinical and sensory anhedonia has already been suggested by Berlin et al. (1998) in the gustatory field. The authors showed that the hedonic responses to sucrose solutions and sweet taste perception threshold might be used as complementary evaluation to quantify anhedonia in depression and schizophrenia.

The purpose of this paper was to confirm the existence of an olfactory anhedonia in depressed patients. We hypothesized that the depressed patients would perceive the pleasant odorant as less pleasant than controls (hedonic aspect). Also, in a binary mixture at iso-intense (equally intense) concentrations of both pleasant and unpleasant odorants, the depressed patients will perceive the pleasant odorants less (qualitative aspect). Moreover they will perceive the pleasant odorant as less intense than the controls, with difficulties in discriminating between different concentrations (quantitative aspect). Furthermore, the olfactory perception of the pleasant odorant should be negatively related to the severity of depression.

\section{Methods}

\subsection{Participants}

Thirty depressive inpatients and 30 control subjects participated in this study. All depressed patients were recruited from the Department of Psychiatry at the University Hospital of Tours, France. All patients scored more than 24 on the Montgomery-Asberg Depression Rating Scale (MADRS; Montgomery and Asberg, 1979). They were investigated $6.8 \pm 6.3$ days after their admission. On the day of the investigation, all patients were being treated with antidepressant and/or anxiolytic drugs. The assessment of psychiatric comorbidities was done with the French version of the Mini International Neuropsychiatric Interview (MINI 5.0; Duburcq et al., 1999; Sheehan et al. 1998). The MINI is a structured interview that allows the clinician to build standardised categorical assessment of the main psychiatric disorders according to the axis I of the DSM- IV. The control subjects were healthy, with no history of mental illness, matched for age and gender with the depressive patients (Table 1). At the time of their recruitment, they were informed that they would have to smell different compounds that can be found in food products. They were given full details about the experimental protocol 
before the beginning of the experiments. The subjects had given their written consent to participate.

Preliminary experiments were carried out with 6 healthy subjects (internal panel), chosen on the basis of their motivation and availability. The exclusion criteria for all participants included possible brain damage, major medical problems, current substance abuse, allergy, a current cold or a problem with their sense of smell. All subjects were selected on the absence of anosmia to the odorants used in the present study.

Table 1. Group characteristics.

\begin{tabular}{lcc}
\hline & MDD patients $(\mathbf{n}=\mathbf{3 0})$ & Controls $(\mathbf{n}=\mathbf{3 0})$ \\
\hline Female / Male Ratio & $12 / 18$ & 12 / 18 \\
Age, Years (SD) & $34.6(11.1)$ & $33.4(9.9)$ \\
Depression & $36.3(6.3)$ & $2.0(2.1)$ \\
$\quad$ MADRS, Score (SD) & $6.7(5.2)$ & - \\
$\quad$ Duration of illness, Months (SD) & $2.1(1.8)$ & - \\
$\quad$ Number of previous episodes (SD) & & - \\
MINI 5.0 & 30 & - \\
MDD, current episode & 11 & - \\
Suicidal risk, last month & - & - \\
(Hypo)-Mania & - & - \\
Panic disorder, agoraphobia & - & - \\
OCD, last month & 3 & - \\
PTSD, last month & 5 & - \\
Alcohol abuse, last 12 months & 2 & - \\
Cannabis abuse, last 12 months & - & - \\
Psychotic disorder, lifetime & - & - \\
Eating disorders, last 3 months & - & \\
GAD, last 6 months & & \\
\hline
\end{tabular}

MDD:Major Depressive Disorder; OCD: Obsessive Compulsive Disorder; PTSD: Post-Traumatic Stress Disorder; GAD: Generalized Anxiety Disorder.

\subsection{Stimuli}

Two odorants with opposite hedonic valences were chosen: vanillin (4-hydroxy-3methoxybenzandehyde), a pleasant odour that evokes mainly happiness (Alaoui-Ismaili et al., 1997a) and butyric acid, described as a rancid odour that induces disgust and anger (AlaouiIsmaili et al., 1997b). Olfactory perception in participants was studied at quantitative (intensity rating and odour intensity discrimination), qualitative (odour identification in binary mixtures) and hedonic levels. The odorants were studied alone (out of mixture) and in binary mixtures.

The odorants were supplied by Fisher Scientific Bioblock, Sigma (Illkirch, France). Three different supra-threshold concentration levels of each odorant were used. They were obtained by multiplication with a constant factor: 10 for vanillin (V) and 5 for butyric acid (B). These factors were selected after a preliminary experiment undertaken on each odorant to choose three concentrations that were approximately iso-intense and easily differentiated by a reduced internal panel (group of assessors chosen to participate in a sensory test) (Table 2).

According to a one-way ANOVA, there was a highly significant concentration effect for both odorants (V: $F=38.44, d f=2, P=0.00, \mathrm{~B}: F=46.90, d f=2, P=0.00$ ). According to NewmanKeuls multiple comparison tests, the subjects were able to discriminate between all 
concentration levels $(\mathrm{C} 1, \mathrm{C} 2, \mathrm{C} 3)$ for both odorants $(P=0.05)$. The iso-intense perception of the different concentrations for both odorants was controlled by using a Student test (B1/V1, $t=-0.54, d f=5, P=0.31 ; \mathrm{B} 2 / \mathrm{V} 2, t=-1.00, d f=5, P=0.18 ; \mathrm{B} 3 / \mathrm{V} 3, t=-1.00, d f=5, P=0.18$ ). (Table 2).

Table 2. Concentrations of the odorant solutions and mean of panel perceived odour intensity (preliminary experiment) of unmixed odorants. Concerning the odour intensity: in line (intensity discrimination), values with the same letters are not significantly different at $\alpha=5 \%$, according to a Newman-Keuls test; in column (iso-intensity), values with the same letters are not significantly different at $\alpha=5 \%$, according to a Student test.

\begin{tabular}{|c|c|c|c|c|c|c|}
\hline \multirow[t]{2}{*}{ Odorant } & \multicolumn{3}{|c|}{ Concentration (mg/l) } & \multicolumn{3}{|c|}{$\begin{array}{l}\text { Mean of panel perceived odour } \\
\text { intensity (preliminary experiment) }\end{array}$} \\
\hline & $\mathrm{C} 1$ & $\mathrm{C} 2$ & $\mathrm{C} 3$ & I1 & $\mathrm{I} 2$ & $\mathrm{I} 3$ \\
\hline Vanillin & 60 & 600 & 6000 & $11.67^{\mathrm{A}}$ & $21.67^{\mathrm{B}}$ & $32.50^{\mathrm{C}}$ \\
\hline Butyric acid & 0.032 & 0.16 & 0.8 & $12.50^{\mathrm{A}}$ & $22.50^{\mathrm{B}}$ & $33.33^{\mathrm{C}}$ \\
\hline
\end{tabular}

The choice of the three concentrations (C1, C2, C3) for both odorants was carried out as following. Several series of odorants with different concentration factors were prepared. We prepared the same second concentration (C2) known as having the same intensity $(600 \mathrm{mg} / \mathrm{l}$ for $\mathrm{V}, 0.16 \mathrm{mg} / \mathrm{l}$ for B). Each series included 3 different concentration levels of the same odorant. These concentrations were chosen because they had a middle intensity. For each series, the $\mathrm{C} 1$ had a low intensity, but the odour was clearly perceived by subjects, who could detect all concentrations. The C3 for both odorants was not very high, which limited the olfactory saturation and the activation of the trigeminal chemosensory system. The series with the lowest concentrations factor were presented first, in random order. The subjects were asked to smell the odour in the flask and to evaluate its intensity (magnitude estimation method; Cain and Drexler, 1974). If they discriminated the three different concentrations of the odorant, the studied factor was selected for the following study. If not, another series of the odorant with a greater concentration factor was presented.

Concerning the magnitude estimation method, vanillin at $600 \mathrm{mg} / \mathrm{l}(\mathrm{C} 2)$ was used with an allocated magnitude value of 20 as reference. This odorant was chosen in order to facilitate the task for the participants and to minimize the cross-olfactory adaptation during the test. The subjects were asked to memorize the reference value (20) and to assign a number relative to the standard stimulus for each new perceived odour. For example, if the current stimulus is twice intense as the standard stimulus it should be called 40 or if it is half intense, it should be called 10. Thus, when judging the samples, the ratio between the numeric response for the reference and the current sample was supposed to reflect the intensity ratio between these samples.

Each experiment comprised 16 stimuli including 3 concentration levels of each odorant (vanillin and butyric acid), their 9 possible combinations and 1 control containing the distilled water. This last stimulus was introduced in order to ensure the reliability of the subject's response and in order to check the absence of the unwanted odours in the solvent. For all experiments, the solutions were made with distilled water. The odorous solutions were poured into $60 \mathrm{ml}$ brown glass flasks (10ml per flask). A three-digit random number coded each flask.

\subsection{Experimental procedure}

Prior to the measurement session, a 20 minute training session took place to familiarize the subjects with odour pleasantness, odour intensity and odour quality rating. The stimuli were 
three different concentrations of both odorants (B1, B2, B3 and V1, V2, V3), and two binary mixtures (B1V1, B1V3). Only two binary mixtures (chosen at random) were used during this training session in order to limit olfactory saturation and tiredness. Subjects had to evaluate the odour pleasantness (hedonic aspect) of the stimulus, to classify the three concentration levels of each odorant in increasing odour intensity, and to evaluate both stimulus intensity (quantitative aspect) and quality (qualitative aspect). The task and the instructions given to the subjects were identical to those given during the measurement sessions and are described below.

The measurement session was carried out during one session of 40 minutes. Each stimulus out of 16 was presented only once. Presentation order was balanced across stimuli and was identical for all subjects. The codes of the flasks were different between the training and measurement session. Earlier experiments (Laing, 1983) showed that each individual optimizes their parameters of sniffing to obtain his maximum sensitivity. Therefore, the subjects were not limited in the time allowed for sniffing, but a 30 second interval between samples was imposed in order to prevent olfactory adaptation.

\subsubsection{Hedonic aspect and familiarity}

Odour familiarity for both odorants (at C2) was evaluated before all tests on a $10 \mathrm{~cm}$ linear scale labelled at each end (unfamiliar odour / very familiar odour), in order to evaluate the influence of the odour's familiarity on its olfactory perception. Firstly, after the familiarization session, the subjects smelled each flask and had to evaluate the pleasantness of the perceived odour stimulus on a $10 \mathrm{~cm}$ linear scale labelled at each end (highly unpleasant / highly pleasant). The resulting response was expressed with a score ranging from 0 to 10 .

\subsubsection{Qualitative aspect}

Secondly, the subjects' task was to identify the perceived odour quality of each stimulus (unmixed odorants or mixtures). Before the measurement session, they were instructed to smell and to memorize the odour quality of two flasks containing vanillin (coded V) and butyric acid (coded B) respectively. The subjects were informed that after this, they would have to identify the memorized odours in several other flasks. They knew that each flask may contain one or both odorants at the same time. If they perceived a different odour, they had to choose the appropriate response and describe the new odour. Thus, subjects could choose one of five responses: "V" if they thought the sample contained only vanillin, "B" if they thought the sample contained only butyric acid, "BV" if they thought both vanillin and butyric acid were present, "nothing" if they thought the sample was water or "another odour" if they perceived a different odour. These last two possible responses were added in order to predict an eventual inhibition phenomenon (no perception of the binary mixture even if the odorants were present at supra-threshold level out of mixture) or to predict the formation of a new odour.

\subsubsection{Quantitative aspect}

As far as the quantitative aspect is concerned, subjects had to evaluate the perceived odour intensity of each odorant. Subjects judged the perceived intensity by the magnitude estimation method. As described in part 2.2, they were instructed to memorize the intensity of the reference (coded REF and corresponding to the vanillin at $\mathrm{C} 2$ ) and then to evaluate the perceived intensity of each flask according to the reference. For this task, the subjects were asked to focus only on the odour intensity, independently from the odorant quality. The subjects were blind to the contents of the flasks. Thus, if they perceived only one odour, they had to evaluate its intensity $\left(\mathrm{I}_{\mathrm{V}}\right.$ or $\left.\mathrm{I}_{\mathrm{B}}\right)$, and if they perceived a mixture of two odorants $(\mathrm{V}, \mathrm{B})$ 
they had to evaluate both $\mathrm{I}_{\mathrm{V}}$ and $\mathrm{I}_{\mathrm{B}}$. In the present paper only the perceived intensities of the unmixed stimuli are treated. In case the subject did not perceive any odour in the flask, they were instructed to not evaluate all characteristics cited above.

The experimental design is schematically presented in supplementary material 1 .

\subsection{Statistical analysis}

All statistical analyses were performed at $\alpha=5 \%$. They were conducted using XLSTAT ${ }^{\circledR}$-Pro, release 5.2.

\subsubsection{Hedonic aspect and familiarity}

The two-tailed unpaired Student test was used for each stimulus to compare the pleasantness response and the odour familiarity level between the two groups of subjects (patients and controls).

\subsubsection{Qualitative aspect}

In order to present the qualitative responses $(\mathrm{B}, \mathrm{V}, \mathrm{BV})$, experimental data were reported on a graph based on two parameters [probability of response $\square=f(\tau)$ ]. Initially, this graph was introduced to predict the binary mixture's quality by knowing the perceived odour intensity of both unmixed odorants (Olsson, 1994). Then, this graphical presentation was used in modelling the experimental data by using the linear logistic model. The advantage of this last model is that it is able to show a dominance of an odorant when both aroma compounds are present at an iso-intense level in the binary mixture (Atanasova et al., 2005).

In the present study, a linear logistic model was used to model the probability of $\mathrm{V}$ (respectively B) responses according to the acid butyric intensity proportion in the mixture, tau $(\tau)$ (Figure 1). Tau $(\tau)$ is the ratio between the perceived intensity of one of the odorants (acid butyric in our case) and the sum of the perceived intensities of each unmixed odorant $[\tau$ $\left.=\mathrm{I}_{\mathrm{B}} /\left(\mathrm{I}_{\mathrm{B}}+\mathrm{I}_{\mathrm{V}}\right)\right]$. It reflects the relative intensity proportion of one odorant, compared to the sum of the intensities of both odorants. When $\tau=0.5$, the mixture is composed of iso-intense unmixed components.

This linear logistic model was fitted using individual numbers of $\mathrm{B}$ (respectively $\mathrm{V}$ ) responses, assumed to be distributed according to binomial distributions. It supposes that the relationship between the logit of $\mathrm{B}$ (respectively $\mathrm{V}$ ) response probability and $\tau$ is linear, and that the intercepts vary according to subjects:

[Equation 1]

$$
\operatorname{logit}\left(\mathrm{p}_{\mathrm{B}}\right)=\log \left(\frac{\mathrm{p}_{\mathrm{B}}}{1-\mathrm{p}_{\mathrm{B}}}\right)=\mu+\alpha_{\text {subject }}+\beta(\tau-0.5)
$$

With the constraint:

$$
\sum_{\text {subjects }} \alpha_{\text {subject }}=0
$$

Number of acid butyric responses $\sim$ binomial distribution $\left(n=1 ; p_{B}\right)$
[Equation 2]

$\operatorname{logit}\left(\mathrm{p}_{\mathrm{v}}\right)=\log \left(\frac{\mathrm{p}_{\mathrm{v}}}{1-\mathrm{p}_{\mathrm{v}}}\right)=\mu^{\prime}+\alpha_{\text {subject }}^{\prime}+\beta^{\prime}(\tau-0.5)$

With the constraint: $\quad \sum_{\text {subjects }} \alpha_{\text {subject }}^{\prime}=0$

Number of vanillin responses $\sim$ binomial distribution $\left(n=1 ; p_{V}\right)$

$\mu$ ( $\mu^{\prime}$ respectively) is the mean panel intercept i.e. the value of logit $\left(\mathrm{p}_{\mathrm{B}}\right)$ at $\tau=0.5$. The intercept for each subject is $\mu+\alpha_{\text {subject }}\left(\mu^{\prime}+\alpha^{\prime}\right.$ subject respectively) and $\beta$ ( $\beta$ ' respectively) is the panel slope.

In order to study the dominance of the odorant, the overlapping of the intercepts' (B and V responses) confidence intervals at $\tau=0.5$ was used. This calculation of the confidence intervals 
(CI) gives an indication of the statistical significance $(\alpha=0.05)$ of the dominance of the one odour over the other. In order to study BV responses, a logistic model [logit $\left.\left(\mathrm{p}_{\mathrm{BV}}\right)\right]$ of three parameters was used: intercept, slope and curvature. The intercept, slope and curvature were not assumed to vary from one subject to another.

$\operatorname{logit}\left(\mathrm{p}_{\mathrm{BV}}\right)=\log \left(\frac{\mathrm{p}_{\mathrm{BV}}}{1-\mathrm{p}_{\mathrm{BV}}}\right)=\mu^{\prime \prime}+\beta^{\prime \prime}(\tau-0.5)+\gamma^{\prime \prime}(\tau-0.5)^{2} \quad$ [Equation 3]

$\mu$ ' is the panel intercept, $\beta$ ', is the panel slope, and $\gamma$ ' is the panel curvature.

The panel intercept was used to determinate the probability of BV responses at iso-intensity of both odorants for both groups of subjects (patients and controls).

In the graphical representation, the probability of giving a B response is plotted against $\tau$, as well as the probability of giving a $\mathrm{V}$ response and the probability of giving a BV response (Figure 1). For B, and $\mathrm{V}$ responses, the effects are sigmoidal, because their probabilities lie between 0 and 1 . For $\mathrm{B}$ and $\mathrm{V}$ responses, the model reflects a linear effect in logit scale, with an intercept depending on the subject and a common slope. If we had represented the logit of response probabilities versus $\tau$, we would have observed parallel straight lines with intercepts varying according to subjects. In Figure 1, only the panel probabilities are represented (parameters $\mu, \beta, \mu$ ', $\beta$ '), giving a unique sigmoidal curve. As far as "BV" responses are concerned, the effect is quadratic on the logit scale, giving an effect such as those shown in Figure 1, on the probability scale.

The procedure in XLSTAT $^{\circledR}$-Pro and the table with the experimental data of depressed subjects used in order to apply the linear logistic model are presented as supplementary material 2.

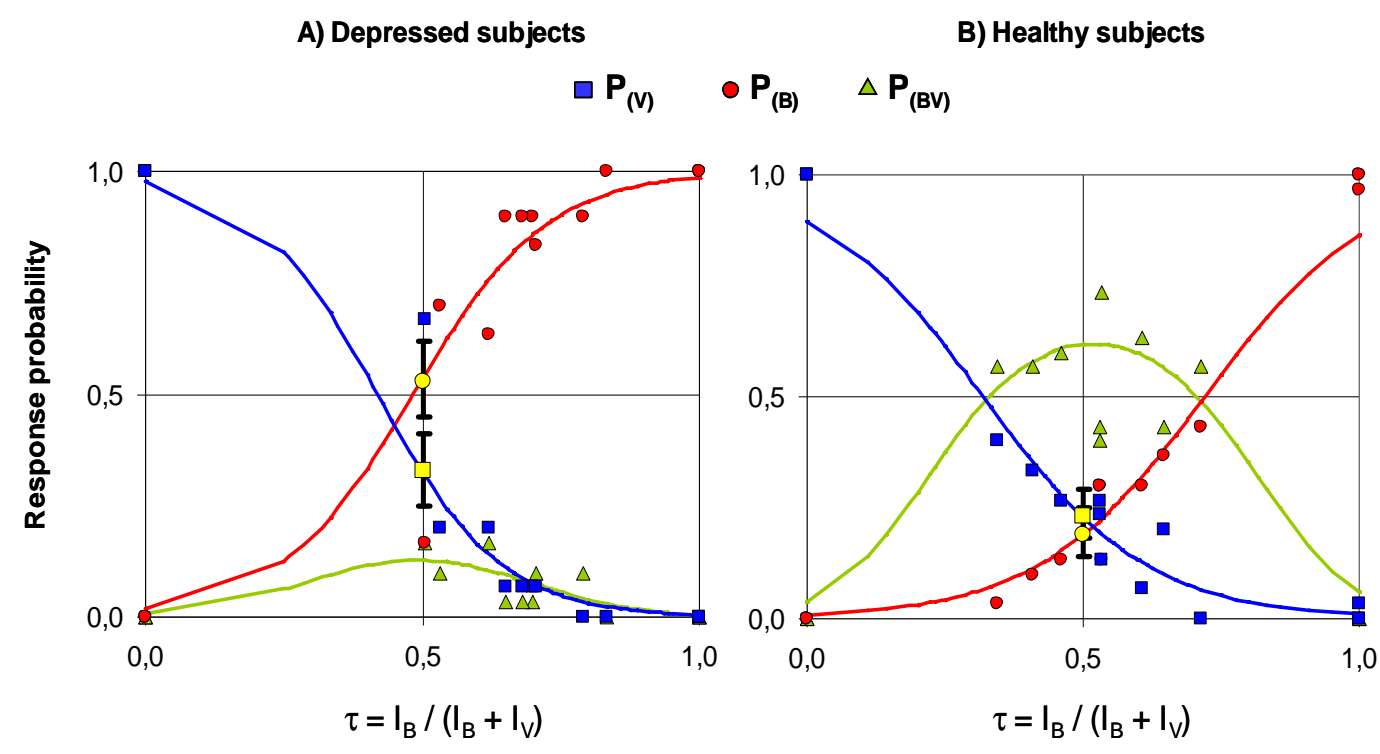

Fig. 1. Qualitative "B", "V" and "BV" experimental response probabilities versus $\tau$. The means values of the subjects of "B" (respectively "V" and "BV") experimental response probabilities are represented with different symbols. Fitted curves represent the response probabilities obtained with linear logistic model. The intercept (at tau=0.5) of " $\mathrm{B}$ " and " $\mathrm{V}$ " responses with $95 \%$ confidence intervals (vertical bars) for both groups of subjects (depressed patients and control subjects) are presented with yellow symbols (circle for "B" and square for "V" responses). 


\subsubsection{Quantitative aspect}

The two-tailed unpaired Student test was used for each unmixed stimulus to compare the perceived odour intensity score of different concentrations between the two groups of subjects (patients and controls).

The effect of odour solution level (unmixed odorants) on intensity scores was tested by oneway analyses of variance, using concentration as factor. Newman-Keuls multiple mean comparison test permitted two-by-two comparisons of the different concentration levels.

\subsubsection{Relation between the score of depression and the hedonic, quantitative and qualitative perception of the stimuli}

In order to study the relationship between the hedonic and the intensity perception of the subject and their level of depression, a Pearson correlation coefficient was calculated between the MADRS score and the hedonic or intensity score of the single odorants.

The Pearson correlation coefficient was also used in order to study the relationship between the MADRS score of depression and the probability of identifying the odour of the vanillin or the butyric acid in the binary mixture when both odorants are present at iso-intensity. The probability of vanillin (respectively butyric acid) responses was presented as the intercept of each subject, obtained from the linear logistic model fitted using individual numbers of each $\mathrm{B}$ and $\mathrm{V}$ responses.

\section{Results}

First of all, considering the perception of the control stimulus, only one depressed subject perceived a very weak unpleasant odour (intensity 2 compared to the reference 20), which he could not describe. All other subjects did not perceive any odour in the flask. Consequently, we can presume that the solvent does not contain any unwanted distracting odours.

\subsection{Hedonic aspect and familiarity}

As expected, in all unmixed stimuli the hedonic scores of pleasant odour were significantly higher than those of unpleasant odours at the three levels of concentration in depressed patients (two-tailed paired Student test; B1/V1: $t=5.02, d f=29, P=0.00 ; \mathrm{B} 2 / \mathrm{V} 2: t=10.12$, $d f=29, P=0.00$ and B3/V3: $t=15.73, d f=29, P=0.00$ ) and in controls (two-tailed paired Student test; B1/V1: $t=6.96, d f=29, P=0.00 ; \mathrm{B} 2 / \mathrm{V} 2: t=15.13, d f=29, P=0.00$ and $\mathrm{B} 3 / \mathrm{V} 3: t=15.39$, $d f=29, P=0.00)$.

All stimuli except one (V2) were perceived as less pleasant by depressed patients. The results showed that this difference is significant for nine out of fifteen stimuli (Figure 2). 


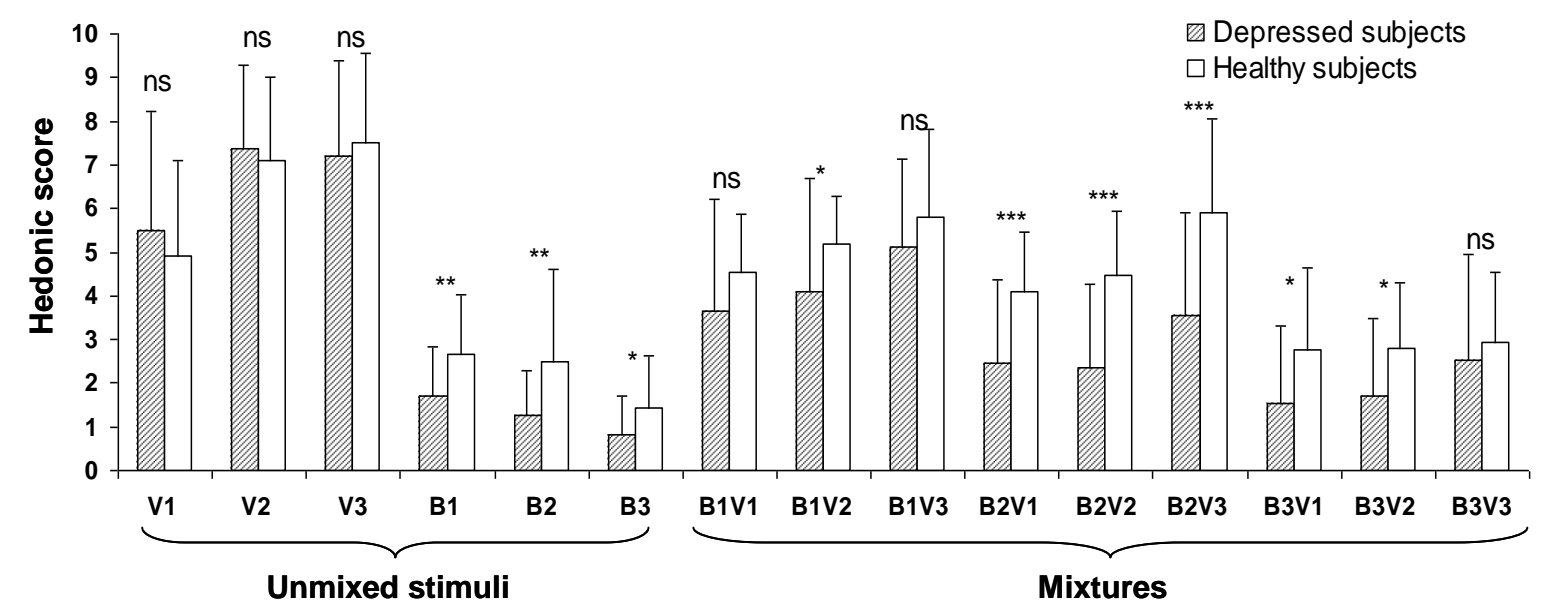

Fig. 2. Mean hedonic score for each stimulus obtained by depressed patients and healthy controls. Vertical bars indicate the standard deviation of the mean. Means comparison (two-tailed unpaired Student test) between depressed subjects and healthy subjects (ns: difference not significant; *: $P<0.05$; **: $P<0.01 ; * * *: P<0.001$ )

The hedonic scores were significantly different between the two groups only for the different concentration levels of $\mathrm{B}$ and not for the $\mathrm{V}$. Thus, the depressed patients judged unpleasant odours more unpleasant than the healthy controls (two-tailed unpaired Student test; for all unpleasant stimuli $d f=58, P<0.05$, Figure 2). No differences between each group was observed for pleasant odours (two-tailed unpaired Student test; for all pleasant stimuli $d f=58$, $P>0.05$, Figure 2). Moreover, there was no relationship between the hedonic scores of each unmixed odorant and the depression scores (MADRS) (Pearson correlation coefficient, for all stimuli $P>0.05)$.

Concerning the mixtures, all stimuli except three (B1V1, B1V3 and B3V3) were perceived as significantly less pleasant by depressed patients (two-tailed unpaired Student test, $d f=58$, $P<0.05$, Figure 2).

There was no significant difference between both groups concerning their judgement of the familiarity of both odorants. For both groups the vanillin was judged as a significantly more familiar odour (two-tailed paired Student test; patients: $t=-15.8, d f=29, P=0.00$; controls $t=-$ 15.6, $d f=29, P=0.00)$.

\subsection{Qualitative aspect}

Considering stimulus quality evaluation, the term "another odour" was never chosen. The answer "nothing" was chosen only two times by healthy subjects, that corresponds to a frequency of use of $<3.3 \%$ in all subjects. This last answer was never chosen by depressed subjects. Consequently the two answers "another odour" and "nothing" were disregarded and only the V, B and BV answers were analysed.

To study odour quality perception, the individual response probabilities of $\mathrm{B}, \mathrm{V}$, and $\mathrm{BV}$ responses were represented versus the relative odorant compound intensity, $\tau$ (Figure 1, symbols). A graphical examination of the depressed subjects' experimental data (Figure 1A, symbols) suggests that for mixtures of iso-intense components, the probability of identifying the mixture as vanilla is smaller than the probability of identifying the mixture as butyric acid $\left(\mathrm{P}_{(\mathrm{V})}<\mathrm{P}_{(\mathrm{B})}\right.$ at $\left.\tau=0.5\right)$. In other words, the relationship between $\mathrm{P}_{(\mathrm{V})}$ and $\tau$, and between $\mathrm{P}_{(\mathrm{B})}$ and $\tau$ are not symmetrical compared to the vertical line at $\tau=0.5$. On the contrary, the results obtained by healthy subjects (Figure 1B, symbols) showed that for mixtures of iso-intense 
components, the probability of identifying the mixture as pleasant is slightly higher than the probability of identifying the mixture as unpleasant $\left[\mathrm{P}_{(\mathrm{V})}>\mathrm{P}_{(\mathrm{B})}\right.$ at $\left.\tau=0.5\right]$.

In order to evaluate the statistical significance of the previously observed trends, a linear logistic model was applied on odour quality experimental data. The response probabilities $\mathrm{P}_{(\mathrm{B})}, \mathrm{P}_{(\mathrm{V})}$ and $\mathrm{P}_{(\mathrm{BV})}$ were modelled separately. The modelling results are presented graphically in Figure 1 (curves). Using the linear logistic model, the hypothesis of no dominant odorant was statistically tested. This hypothesis implies an equality of B and V response probabilities for mixtures of iso-intense components $(\tau=0.5)$.

The depressed subjects' results showed that the $95 \%$ CI of the panel intercept of the two odorants did not overlap (Figure 1, yellow symbols). More precisely, when unmixed odorants were present at iso-intense levels $(\tau=0.5)$, the mean probability of identifying the mixture as vanilla was smaller than the mean probability of identifying the mixture as butyric acid $\left(\mathrm{P}_{(\mathrm{V})}<\mathrm{P}_{(\mathrm{B})}, \tau=0.5\right)$. Obviously, this finding implied that the curves were not symmetrical with respect to $\tau=0.5$ (Figure 1A). Moreover, the MADRS scores correlated negatively with the probability to identify the vanilla odour $(r=-0.395, P=0.019)$ and positively with the probability to identify the butyric acid odour $(r=0.481, P=0.005)$ in the iso-intense mixture.

The controls' results showed that the mean probability of answering B was $0.19(\tau=0.5)$ whereas the mean probability of answering V was 0.23 at the same $\tau$ value (Figure 1B). This difference is moderate and not significant, because the $95 \% \mathrm{CI}$ of the panel intercept of the two odorants overlap, i.e. there is no qualitative dominance of one or other odour (Figure 1, yellow symbols).

The probability of identifying both pleasant and unpleasant components $\left(\mathrm{P}_{(\mathrm{BV})}\right)$ in the isointense mixture was five times higher in healthy subjects $\left(\mathrm{P}_{(\mathrm{BV})}=0.61\right)$ compared to depressed subjects $\left(\mathrm{P}_{(\mathrm{BV})}=0.12\right)$ (Figure 1). This finding indicates that pleasant and unpleasant sensations elicited by the $\mathrm{B} / \mathrm{V}$ mixture are more distinct and easier to recognize for healthy subjects than depressed subjects. In patients, most of the subjects were able to identify only one of the two odours, whatever the mixture proportion and even in mixtures of iso-intense components.

\subsection{Quantitative aspect}

\subsubsection{Discrimination of the stimulus concentration levels}

The results of the one-way ANOVA showed a highly significant concentration effect in patients for vanillin $(F=31.16, d f=2,87, P=0.00)$ and for butyric acid $(F=16.38, d f=2,87$, $P=0.00)$. The same level of significance for the concentration effect was also observed in control subjects for $\mathrm{V}(F=18.28, d f=2,87, P=0.00)$ and $\mathrm{B}(F=25.98, d f=2,87, P=0.00)$. The results of the Newman-Keuls multiple comparison tests showed that healthy controls were able to discriminate all concentration levels of both odorants $(\alpha=0.05)$ (Table 3). Depressed patients discriminated the different concentration levels only for the unpleasant component. For pleasant components, they classified the last two concentration levels in the same group (Table 3). The patients perceived the odour intensity of all unpleasant stimuli as significantly more intense and the odour intensity of all pleasant odours as significantly less intense in comparison to control subjects (two-tailed unpaired Student test, for all stimuli $d f=58$, $P<0.05$ ) (Figure 3). 
Table 3. Mean odour intensity ratings for both odorants and for both groups of subjects (depressed patients and controls).

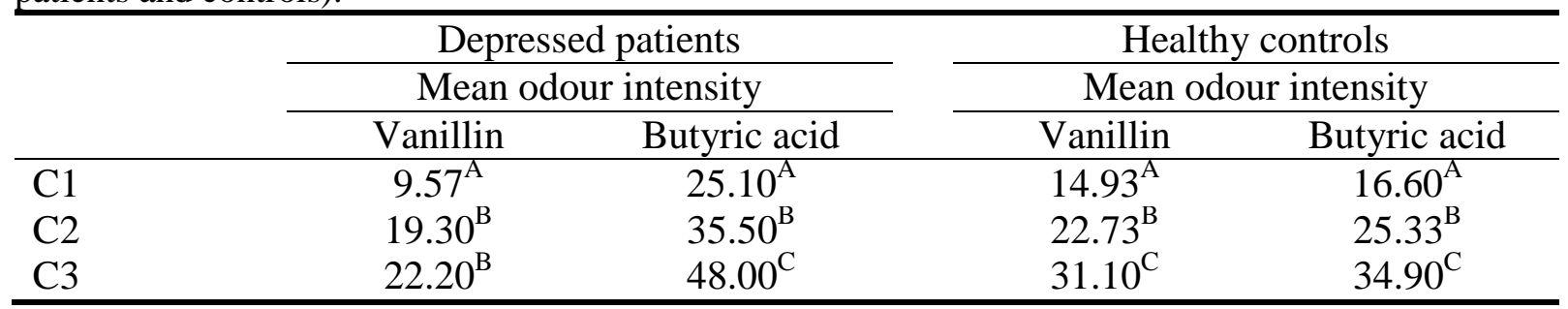

For each group and for each odorant, values with the same letters are not significantly different at $\alpha=5 \%$, according to a Newman-Keuls test.

The calculation of the Pearson correlation coefficients showed that MADRS scores were not correlated with the intensity score of each unmixed stimulus odorant (B1, B2, B3, V1, V2 and V3) (for all stimuli $P>0.05$ ).

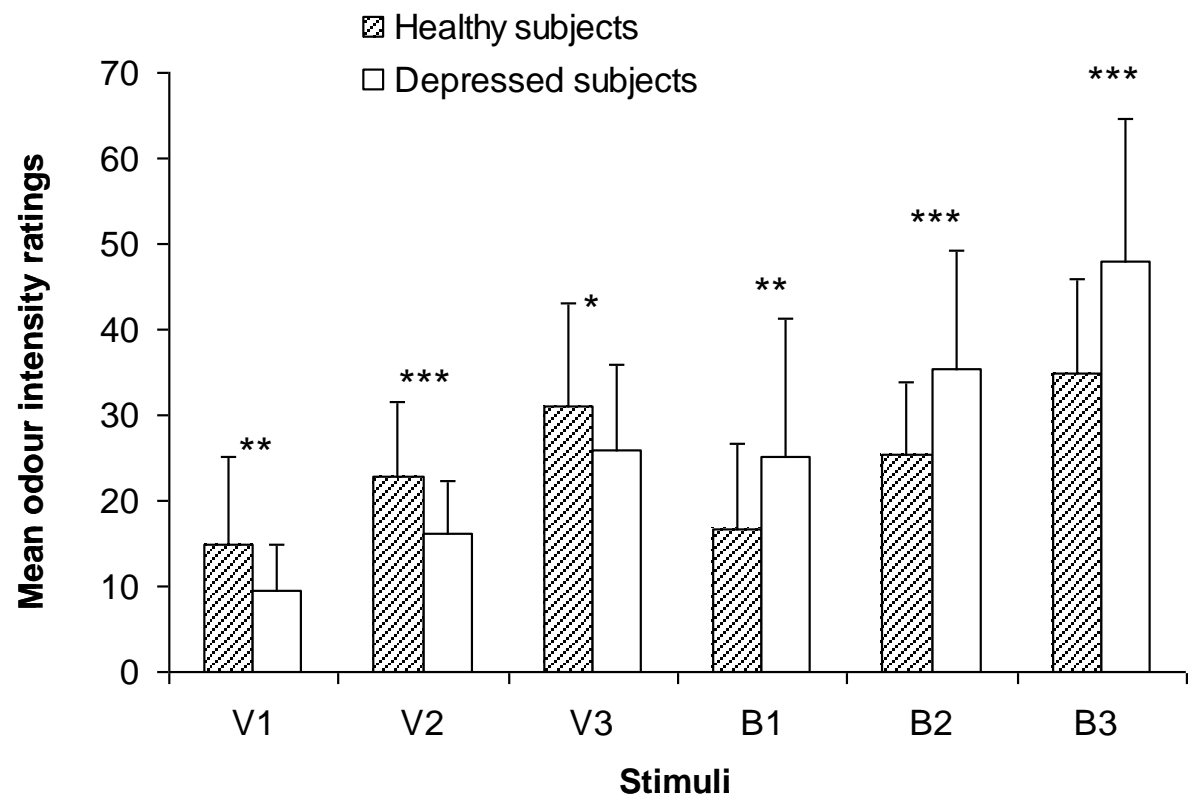

Fig. 3. Mean odour intensity ratings for each unmixed stimulus obtained by depressed patients and healthy controls. Vertical bars indicate the standard deviation of the mean. Means comparison (two-tailed unpaired Student test) between depressed subjects and healthy subjects $(*: P<0.05 ; * *: P<0.01 ; * * *$ : $P<0.001)$.

\section{Discussion}

The aim of the present study was to assess the presence of an olfactory anhedonia in depressed patients by studying olfactory perceptions at three different levels: pleasantness, intensity and quality. Therefore, these characteristics were studied using two odorants with opposite valence (vanillin and butyric acid). We hypothesized that depressed patients would display an impaired olfactory perception of the pleasant odorant only, compared to the controls. 
Concerning the hedonic perception of odorants, we expected that the depressed patients would perceive the pleasant stimuli as less pleasant compared to controls. The results showed a difference between the two groups of subjects only for the unpleasant unmixed stimuli, which were perceived as more unpleasant by patients. This suggests the presence of an olfactory based cognitive bias or to a negative olfactory alliesthesia. Indeed, negative alliesthesia corresponds to an increased perception of the negative aspects of a stimulus. The alliesthesia observed in the depressed subjects could be related to the selective processing of negative information shown by these subjects who are known for making dysfunctional attributions, and for engaging in more negative automatic thinking.

Lombion-Pouthier et al. (2006) and Pause et al. (2001) studies were the only ones that evaluated the hedonic ratings for several odours in psychiatric disorders. Selecting only pleasant odorants, the first study showed that depressed patients over-evaluated the pleasantness. The authors found these ratings surprising as the depressed subjects usually experienced negative emotional cues and their suggested explanation was that of a functional consequence of brain processes underlying depressive states (Lombion-Pouthier et al., 2006). These alterations could be related to dysfunctions in the orbitofrontal cortex, a brain area associated with the hedonic evaluation of odours (Savic, 2001). Indeed, its activity seems increased in depressive states (Drevets, 1998; Pause et al., 2003). The second study, carried out by Pause et al. (2001) observed a trend at difference for the valence ratings of one odour (citral), which was perceived as more pleasant by the depressive and not by the control subjects; this was explained by the relaxing properties of the lemon-like odour. The results of the present study on the hedonic mixtures perception showed that most of the mixtures were perceived as significantly less pleasant by depressed patients. These results suggest that in binary mixtures of both pleasant and unpleasant odorants at different proportions, the depressed subjects perceived unpleasant odours better. Taking into account all the results cited above, we can suppose that the hedonic odour perception at depressed subjects depends on the functional consequences of the brain processes underlying depressive states, but also on the odorants' characteristics - their relaxing effects and their emotional subjective influence on the subject.

Regarding the quantitative odour perception (intensity ratings and discrimination of the different intensity levels), we expected that the depressed patients would perceive the pleasant stimuli as less intense compared to controls and they would not be able to discriminate between its three different concentration levels. The results showed that patients perceived as more intense unpleasant stimuli and as less intense pleasant stimuli compared to the controls. Indeed, we can summarize that olfactory anhedonia was observed for pleasant odorant and olfactory alliesthesia for unpleasant odorant. On the contrary, no significant difference in intensity ratings between patients and controls was found in previous studies (Pause et al., 2001; Thomas et al., 2002). An explanation of this difference could be the type of the studied odorants, the severity of the subjects' depression and the methodology (type of scales, experimental conditions, etc.). As for the odour intensity discrimination, patients discriminated the different concentration levels only for the unpleasant component, but not of the vanillin. Indeed, these results suggest the presence of an olfactory anhedonia in depressed subjects at a quantitative level.

As to the qualitative aspect, we hypothesized that at iso-intense levels of both pleasant and unpleasant odorants in a binary mixture, the depressed patients would perceive less the odour of the pleasant component compared to the controls. The experimental data of the patients 
highlighted that when both odorants are present at iso-intense levels in the mixture, the probability of identifying the unpleasant odorant is significantly higher than the probability of identifying the pleasant odorant, whereas controls perceive both odorants in the mixture. These results show the presence of an olfactory anhedonia at a qualitative level also. Additional correlative analyses revealed that the olfactory qualitative perception of a binary mixture could be predicted by the depression scores: the more one subject is depressive, the more he perceives the unpleasant stimulus in the binary iso-intense mixture, and on the contrary, the more he is depressed, the less he perceives the pleasant stimulus.

Results from a recent study showed that unpleasant odorants are judged more quickly than pleasant odorants (Bensafi et al., 2002). This difference in temporal treatment could obviously be a basis for the dominance of a quality in mixtures of iso-intense components, but in this case, the unpleasant odour should dominate. However in our study, both odorants were perceived by controls and dominance of the unpleasant odorant was observed for the patients only. Another factor, which could influence the perception of the mixture's constituents, is the odour familiarity. Rabin (1988) showed that a familiar odour is more easily perceived and discriminated than an unfamiliar odour. The familiarity evaluation results of the two studied odorants showed that for both groups the vanillin was judged as a significantly more familiar odour, but this odorant was not perceived in the same manner by both groups of subjects. Consequently we cannot explain the observed results with this cognitive factor. Another possible explanation of the observed trends could be the eventual activation of different central structures according to the trigeminal proprieties of the studied odorants. It is know that the olfactory nerve-mediated odorants and combined olfactory and trigeminal nervemediated odorants activate different regions in the brain. Trigeminal stimulating odours have been shown to produce bilateral brain activation (Yousem et al., 1997). In the present study, the highest concentration of both odorants was chosen to be below their trigeminal threshold. But this evaluation was carried out with the internal trained panel. In order to exclude this possible influence on the subjects' olfactory perception, it would be necessary to evaluate the trigeminal level of the odorants with the participants (patients and controls) of the study.

Olfactory dysfunction has been characterized according to whether the deficit is peripheral or central in nature. Decreased olfactory acuity in the absence of any other olfactory dysfunction has been thought to be associated with peripheral alteration. Deficits in olfactory identification, discrimination, recognition, and memory, in the presence of intact acuity imply alteration of more central brain processing systems (Martzke et al., 1997). Our results showed that depressed subjects perceived the positive stimulus less, from a discrimination and identification point of view. These findings suggest that there is an olfactory dysfunction of central brain processing systems.

During the last 10 years, the application of modern brain imaging techniques (positron emission tomography) has shown that odour perception and emotions are processed in similar brain structures (Zald and Pardo, 2000; Lane et al., 1997). Zatorre et al. (1992) outlined the importance of the piriform, orbitofrontal and insular cortex in human odour perception. Focusing on the emotional value of olfactory stimulation, several authors found the amygdala to be activated when aversive or novel odours were presented (Small et al., 1997; Zald and Pardo, 1997). An abnormal activation in the amygdala and the orbitofrontal region were confirmed in depressive patients in several studies (Drevets et al., 1992; Lesser et al., 1994; Brody et al., 1999). Thus, we can suppose that the decreased olfactory perception of the positive stimuli and the better olfactory perception of the negative stimuli of depressive patients are dependent of these neural correlates and with abnormal limbic function. 
Focusing on the emotional evaluation of odours, several authors found that depressive patients differed from controls (Steiner et al., 1993; Pause et al., 2000). The present study demonstrated that altered olfactory perception in depressed patients depends on the odours hedonic valence (and consequently to the generated emotion). This may be related to alteration at the affective level. Indeed, depression is characterized by an alteration of processing of the affective aspects related to odour perception (olfactory bias toward negative odours: olfactory negative alliesthesia or olfactory bias toward positive odours: olfactory anhedonia). This alteration may be related to anhedonia, a well-known depression symptom corresponding to a disturbed experience of pleasure, i.e. a gradual loss of the ability to experience physical or psychic pleasure. The results of the present study demonstrating an olfactory anhedonia suggest that anhedonia may be observed using methods other than questionnaires (Fawcett et al., 1983). An interesting theoretical question is whether olfactory anhedonia and olfactory negative alliesthesia are a state or a trait cognitive marker. Evaluating this requires two complementary measurements: one before any psychiatric treatment, and one during a follow-up investigation a few months after remission.

In summary, with the present study we could validate our hypothesis of the existence of an olfactory anhedonia in depression at qualitative (odour identification) and quantitative (intensity discrimination) perceptual levels only. We also observed an olfactory negative alliesthesia related to the odours hedonic valence and the perceived odour intensity. These observations need to be confirmed by further studies using other olfactory stimuli with different pleasantness level, which evoke different positive and negative emotions.

\section{Acknowledgements}

The authors would like to thank Fatima Zahra Ghayout, Sandrine Auberval and ClaudeEmmanuelle Fausther for their very helpful assistance for the sensory tests with controls. The authors thank Philip Brittain (Institute of Psychiatry, London, UK) for the final English revision of the text.

\section{References}

Alaoui-Ismaili, O., Robin, O., Rada, H., Dittmar, A., Vernet-Maury, E., 1997a. Basic emotions evoked by odorants: comparison between autonomic responses and selfevaluation. Physiology and Behavior 62, 713-720.

Alaoui-Ismaili, O., Vernet-Maury, E., Dittmar, A., Delhomme, G., Chanel, J., 1997b. Odor hedonics: connection with emotional response estimated by autonomic parameters. Chemical Senses 22, 237-248.

Atanasova, B., Thomas-Danguin, T., Chabanet, C., Langlois, D., Nicklaus, S., Etiévant, P., 2005. Perceptual Interactions in Odour Mixtures: Odour Quality in Binary Mixtures of Woody and Fruity Wine Odorants. Chemical Senses 30, 209-217.

Bensafi, M., Rouby, C., Farget, V., Vigouroux, M., Holley, A., 2002. Asymmetry of pleasant vs. unpleasant odor processing during affective judgment in humans. Neuroscience Letters 328, 309-313.

Berlin, I., Givry-Steiner, L., Lecrubier Y., Puech, A.J., 1998. Measures of anhedonia and hedonic responses to sucrose in depressive and schizophrenic patients in comparison with healthy subjects. European Psychiatry 13, 303-309.

Brand, G., 1999. La latéralisation olfactive chez l'homme. Clinical Neurophysiology 29, 495506.

Brody, A.L., Saxena, S., Silverman, D.H.S., Alborzian, S., Fairbanks, L.A., Phelps, M.E., Huang, S.C., Wu, H.M., Maidment, K., Baxter, L.R., 1999. Brain metabolic changes in 
major depressive disorder from pre- to post-treatment with paroxetine. Psychiatry Research: Neuroimaging Section 91, 127-139.

Broughan, C., 2002. Odours, emotions, and cognition - how odours may affect cognitive performance. International Journal of Aromatherapy 12, 92-98.

Cain, W.S. and Drexler, M. (1974) Scope evaluation of odor counteraction and masking. Annals New York Academy of Sciences. 237, 427-439.

Drevets, W.C., 1998. Functional neuroimaging studies of depression: the anatomy of melancholia. Annual Review of Medicine 49, 341-361.

Drevets, W.C., Videen, T.O., Price, J.L., Preskorn, S.H., Carmichael, S.T., Raichle, M.E., 1992. A functional anatomical study of unipolar depression. The Journal of Neuroscience $12,3628-3641$.

Duburcq, A., Blin, P., Charpak, Y., Blachier, C., Allicar, M.P., Bouhassira, M., Hergueta, T., Lecrubier, Y., 1999. Use of a structured diagnostic interview to identify depressive episodes in an epidemiologic study: a posteriori internal validation. Revue Epidémiologie et de Sante Publique 47, 455-463.

Ehrlichman, H., Bastone, L., 1992. Olfaction and emotion. In: Serby, M. and Chobor, K.L. (Eds.), Science of Olfaction. Springer-Verlag, New York, pp. 410-438.

Gilbert, A.N., Knasko, S.C., Sabini, J., 1997. Sex differences in task performance associated with attention to ambient odor. Archives of Environmental Health 52, 195-199.

Klein, D.F., 1974. Endogenomorphic depression. A conceptual and terminological revision.Archives of General Psychiatry 31, 447-454.

Laing, DG., 1983. Natural sniffing gives optimum odour perception for humans. Perception $12,99-117$.

Lane, R.D., Reiman, E.M., Bradley, M.M., Lang, P.J., Ahern, G.L., Davidson, R.J., Schwartz, G.E., 1997. Neuronatomical correlates of pleasant and unpleasant emotion. Neuropsychologia 35, 1437-1444.

Lesser, I.M., Mena, I., Boone, K.B., Miller, B.L., Mehringer, C.M., Wohl, M., 1994. Reduction of cerebral blood flow in older depressed patients. Archives of General Psychiatry 51, 677-686.

Lombion-Pouthier, S., Vandel, P., Nezelof, S., Haffen, E., Millot, J.L., 2006. Odor perception in patients with mood disorders. Journal of Affective Disorders 90, 187-191.

MacLeod, A.K., Byrne, A., 1996. Anxiety, depression, and the anticipation of future positive and negative experiences. Journal of Abnormal Psychology 105, 286-289.

Martzke, J.S., Kopala, L.C., Good, K.P., 1997. Olfactory dysfunction in neuropsychiatric disorders: review and methodological considerations. Biological Psychiatry 42, 721-732.

Mathews, A., MacLeod, C., 1994. Cognitive approaches to emotion and emotional disorders. Annual Review of Physiology 45, 25-50.

Mesulam, M.M., 1998. From sensation to perception. Brain 121, 1013-1052.

Millot, J.L., Brand, G., 2001. Effects of pleasant and unpleasant ambient odors on human voice pitch. Neuroscience Letters 297, 61-63.

Montgomery, S.A., Asberg, M., 1979. A new depression scale designed to be sensitive to change. British Journal of Psychiatry 134, 382-389.

Nagai, M., Wada, M., Usui, N., Tanaka, A., Hasebe, Y., 2000. Pleasant odors attenuate the blood pressure increase during rhythmic handgrip in humans. Neuroscience Letters 289, 227-229.

Olsson, M.J., 1994. An interaction model for odor quality and intensity. Perception and Psychophysics 55, 363-372.

Pause, B.M, Raack, N., Sojka, B., Göder, R., Aldenhoff, J.B., Ferstl, R., 2003. Convergent and divergent effects of odors and emotions in depression, Psychophysiology 40, 209-225. 
Pause, B.M., Miranda, A., Göder, R., Aldenhoff, J.B., Ferstl, R., 2001. Reduced olfactory performance in patients with major depression. Journal of Psychiatric Research 35, 271-277.

Pause, B.M., Miranda, A., Nysterud, M., Ferstl, R., 2000. Olfactory and emotional stimulus evaluation in patients with major depression. Zeitschrift für Klininische Psycholologie und Psychotherapie 29, 16-23.

Phillips, M.L., Drevets, W.C., Rauch, S.L., Lane, R., 2003. Neurobiology of emotion perception I: The neural basis of normal emotion perception. Biological Psychiatry 54, 504514.

Rabin, M.D., 1988. Experience facilitates olfactory quality discrimination. Perception and Psychophysics 44, 532-540.

Royet, J.P., Plailly, J., Delon-Martin, C., Kareken, D.A., Segebarth, C., 2003. fMRI of emotional responses to odors: influence of hedonic valence and judgment, handedness, and gender. NeuroImage 20, 713-728.

Rupp, C., Ilmberger, J., Oberbauer, H., Scholz, A., Wanko, C., Hinterhuber, H., 1997. Olfactory deficits in schizophrenia and major depression. In: Int. Symp. Olfaction and Taste XII and A ChemS XIX, July 7-12, 1997, San Diego, CA, pp. 180.

Savic, I., 2001. Processing of odorous signals in humans. Brain Research Bulletin 54, 307312.

Schiffman, S.S., Sattely-Miller, E.A., Suggs, M.S., Graham, B.G., 1995a. The effect of pleasant odors and hormone status on mood of women at midlife. Brain Research Bulletin $36,19-29$.

Schiffman, S.S., Suggs, M.S., Sattely-Miller, E.A., 1995b. Effect of pleasant odors on mood of males at midlife: comparison of African-American and European-American men. Brain Research Bulletin 36, 31-37.

Serby, M., Larson, P., Kalkstein, D., 1990. Olfactory sense in psychoses. Biological Psychiatry 28, 830.

Sheehan, D.V., Lecrubier, Y., Sheehan, K.H., Amorim, P., Janavs, J., Weiller, E., Hergueta, T., Baker, R., Dunbar, G.C., 1998. The Mini-International Neuropsychiatric Interview (M.I.N.I.): the development and validation of a structured diagnostic psychiatric interview for DSM-IV and ICD-10. Journal of Clinical Psychiatry 59, Suppl 20, 22-33.

Small, D.M., Jones-Gotman, M., Zatorre, R.J., Petrides, M., Evans, A.C., 1997. Flavor processing: more than the sum of its parts. NeuroReport 8, 3913-7.

Steiner, J.E., Lidar-Lifschitz, D., Perl, E., 1993. Taste and odor: reactivity in depressive disorders, a multidisciplinary approach. Perceptual and Motor Skills 77, 1331-1346.

Thomas, H.J., Fries, W., Distel, H., 2002. Evaluation of olfactory stimuli by depressed patients. Nervenarzt 73, 71-77.

Wright, J.H., Beck, A.T., 1983. Cognitive therapy of depression: theory and practice. Hosp Community Psychiatry 34, 1119-1127.

Yousem, D.M., Williams, S.C.R., Howard, R.O., Andrew, C., Simmons, A., Allin, M., Geckle, R.J., Suskind, D., Bullmore, E.T., Brammer M.J., Doty, R.L., 1997. Functional MR imaging during odor stimulation: preliminary data. Radiology 204, 833-838.

Zald, D.H., Pardo, J.V., 1997. Emotion, olfaction, and the human amygdala: amygdala activation during aversive olfactory stimulation. Proceedings of the National Academy of Sciences, USA 94, 4119-4124.

Zald, D.H., Pardo, J.V., 2000. Functional neuroimaging of the olfactory system in humans. International Journal of Psychophysiology 36, 165-181.

Zatorre, R.J., Jones-Gotman, M., Evans, A.C., Meyer, E., 1992. Functional localization and lateralization of human olfactory cortex. Nature 360, 339-340.

Zatorre, R.J., Jones-Gotman, M., Rouby, C., 2000. Neural mechanisms involved in odor pleasantness and intensity judgments. Neuroreport 11, 2711-2716. 